\title{
Nonlinear diffraction from a virtual beam
}

Saltiel, Solomon M.; Neshev, Dragomir N.; Krolikowski, Wieslaw; Voloch-Bloch, Noa; Arie, Ady; Bang, Ole; Kivshar, Yuri S.

Published in:

Physical Review Letters

Link to article, DOI:

10.1103/PhysRevLett.104.083902

Publication date:

2010

Document Version

Publisher's PDF, also known as Version of record

Link back to DTU Orbit

Citation (APA):

Saltiel, S. M., Neshev, D. N., Krolikowski, W., Voloch-Bloch, N., Arie, A., Bang, O., \& Kivshar, Y. S. (2010). Nonlinear diffraction from a virtual beam. Physical Review Letters, 104(8), 083902.

https://doi.org/10.1103/PhysRevLett.104.083902

\section{General rights}

Copyright and moral rights for the publications made accessible in the public portal are retained by the authors and/or other copyright owners and it is a condition of accessing publications that users recognise and abide by the legal requirements associated with these rights.

- Users may download and print one copy of any publication from the public portal for the purpose of private study or research.

- You may not further distribute the material or use it for any profit-making activity or commercial gain

- You may freely distribute the URL identifying the publication in the public portal

If you believe that this document breaches copyright please contact us providing details, and we will remove access to the work immediately and investigate your claim 


\title{
Nonlinear Diffraction from a Virtual Beam
}

\author{
Solomon M. Saltiel, ${ }^{1,2, *}$ Dragomir N. Neshev, ${ }^{1}$ Wieslaw Krolikowski, ${ }^{1}$ Noa Voloch-Bloch, ${ }^{3}$ Ady Arie, ${ }^{3}$ \\ Ole Bang, ${ }^{4}$ and Yuri S. Kivshar ${ }^{1}$ \\ ${ }^{1}$ Nonlinear Physics Center and Laser Physics Center, Research School of Physics and Engineering, Australian National University, \\ Canberra ACT 0200, Australia \\ ${ }^{2}$ Department of Quantum Electronics, Faculty of Physics, Sofia University "St. Kliment Ohridski," Bulgaria \\ ${ }^{3}$ School of Electrical Engineering, Faculty of Engineering, Tel-Aviv University, Tel-Aviv, Israel \\ ${ }^{4}$ DTU Fotonik, Department of Photonics Engineering, Technical University of Denmark, 2800 Kongens Lyngby, Denmark
}

(Received 4 December 2009; published 23 February 2010)

\begin{abstract}
We observe experimentally a novel type of nonlinear diffraction in the process of two-wave mixing on a nonlinear quadratic grating. We demonstrate that when the nonlinear grating is illuminated simultaneously by two noncollinear beams, a second-harmonic diffraction pattern is generated by a virtual beam propagating along the bisector of the two pump beams. The observed diffraction phenomena is a purely nonlinear effect that has no analogue in linear diffraction.
\end{abstract}

DOI: 10.1103/PhysRevLett.104.083902

PACS numbers: 42.65.Ky, 42.25.Fx

When a monochromatic wave passes through a spatially periodic medium, efficient linear diffraction occurs for the waves satisfying the Bragg condition. In this linear wave phenomenon, the diffracted wave vector is determined by the vectorial condition, $\vec{k}_{\text {diff }}=\vec{k}_{1}+m \vec{G}_{0}$, where $\vec{k}_{1}$ is the incident wave vector, $\vec{G}_{0}$ is the reciprocal lattice vector, and $m$ is an integer number. If the periodic medium is sufficiently thin, one may also observe the well-known in optics Raman-Nath diffraction. This type of diffraction is governed by the scalar condition $\left|\vec{k}_{\text {diff }}\right| \sin \alpha_{m} \approx m G_{0}$, with $\alpha_{m}$ being the angle between the wave vectors of the incident and diffracted waves. While the concept of Bragg and Raman-Nath diffraction has been considered mostly in the context of propagation of electromagnetic waves [1], it also applies to atom optics [2] and matter waves [3,4].

If the medium has the periodicity in its nonlinear susceptibility, similar diffraction phenomena can also be observed for the harmonics of the fundamental waves. For example, when a laser light interacts with a quadratic nonlinear grating, the emission of a second-harmonic $(\mathrm{SH})$ frequency $2 \omega$ (with a wave vector $\vec{k}_{2}$ ) is observed, giving birth to the nonlinear Bragg diffraction satisfying the condition $\vec{k}_{2}=2 \vec{k}_{1}+m \vec{G}_{0}$ [5-7], as well as the nonlinear Raman-Nath diffraction, satisfying the condition $\left|\vec{k}_{2}\right| \sin \alpha_{m} \approx m G_{0}$ [8]. These two phenomena represent nonlinear analogues of the well-known linear diffraction of waves on a dielectric grating (of a period $\Lambda$, direction of modulation $\vec{s}$, and $\vec{G}_{0}=2 \pi \vec{s} / \Lambda$ ).

In this Letter, we reveal and verify experimentally the existence of a novel type of nonlinear diffraction (NLD) that does not have a linear analogue. This effect is observed when two noncollinear copolarized fundamental beams intersect inside a medium with a spatial modulation of the sign of second-order nonlinear susceptibility $\left(\chi^{(2)}\right)$. Interaction of the fundamental waves mediated by the nonlinear quadratic grating results in a diffraction pattern of the SH which appears as being generated by a virtual (nonexistent) fundamental beam, incident at the sample along the bisector of the two fundamental beams. The study of this new type of NLD, for both one- and twodimensional quadratic nonlinear structures shows that the generation of the $\mathrm{SH}$ is defined solely by the transverse phase matching of the nonlinear process.

In our experiments (Fig. 1), we employ a onedimensional quadratic grating in lithium niobate and a two-dimensional annular structure fabricated in stoichiometric lithium tantalate (SLT). Our laser source is a regenerative amplifier, delivering $7 \mathrm{ps}$ pulses at $1053 \mathrm{~nm}$ [8]. The beam is mildly focused to a size of $\sim 100 \mu \mathrm{m}$ and is split by a biprism of Fresnel made of BK7 glass with the roof angle of $160^{\circ}$. This biprism allows us to produce two fundamental beams intersecting at an internal angle of $2 \gamma=4.56^{\circ}$ (in lithium niobate). This corresponds to an angle $2 \gamma_{2 \omega}=10.78^{\circ}$ between the output non-phase- (a)

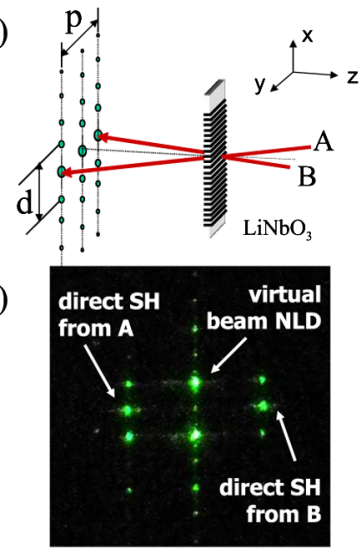

(b)

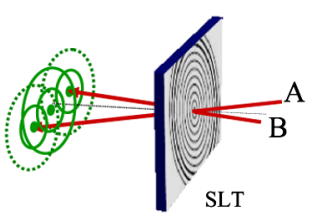

(d)

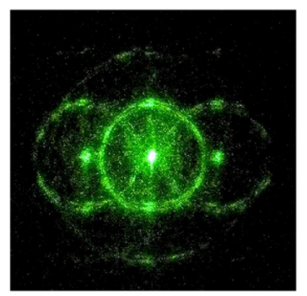

FIG. 1 (color online). Schematic of the experiments with (a) one-dimensional and (b) annular periodically poled grating. (c),(d) Experimentally recorded NLD patterns with two fundamental beams, for the corresponding structures. 
matched SH beams. The bisector of the two pumps is always along the $Z$ axis of the crystal. As seen below, the use of a biprism allows us to calibrate accurately the pattern and to determine the period of the nonlinear grating. In addition, the biprism assures an exact temporal overlap of the pulses carried by both beams. The lithium niobate sample incorporates several periodically poled one-dimensional gratings with a period ranging from $\Lambda=$ 14.1 to $14.9 \mu \mathrm{m}$ and duty cycle close to $50 \%$. The sample is $0.5 \mathrm{~mm}$ thick, with all sides polished. The annular structure $[9,10]$ has a period $\Lambda=7.5 \mu \mathrm{m}$ and a thickness of $0.49 \mathrm{~mm}$.

Two images of the observed nonlinear diffraction patterns are shown in Figs. 1(c) and 1(d). In Fig. 1(c), three distinct diffraction patterns coming from the onedimensional grating are clearly seen. Two of them arise from the individual pump beams $(A$ and $B)$ and the third one (in the middle) appears only if the two fundamental beams cross inside the sample. Figure 2 depicts the diffraction patterns generated without the biprism [Fig. 2(a)], i.e., for a single incident pump, with the biprism located such that both fundamental beams cross outside the sample [Fig. 2(b)], or with the biprism placed at $0.5 \mathrm{~mm}$ from the sample, ensuring overlap of the fundamental beams inside [Fig. 2(c)]. The two pairs of strong SH spots located at the far sides of every pattern correspond to the longitudinally phase-matched SH signal (Čerenkov-type phase matching) $[11,12]$. These two spots arise due to the two different types of $\mathrm{SH}$ interactions in the nonlinear crystal, $O_{2}-O_{1} O_{1}$ and $E_{2}-O_{1} O_{1}$, where $O$ stands for an ordinary wave and $E$ for an extraordinary wave.

Each individual beam induces a NLD pattern with the intensity maxima derived from the formula $\sin \beta_{m}=$ $m \lambda_{2} / \Lambda,\left(\beta_{m}\right.$ is the external diffraction angle). The most interesting is the observation that the two beams can create a third diffraction pattern, without an analogy with linear optics. Indeed, the middle lines in Figs. 1(c) and 2(c) represent a new type of nonlinear diffraction. The two

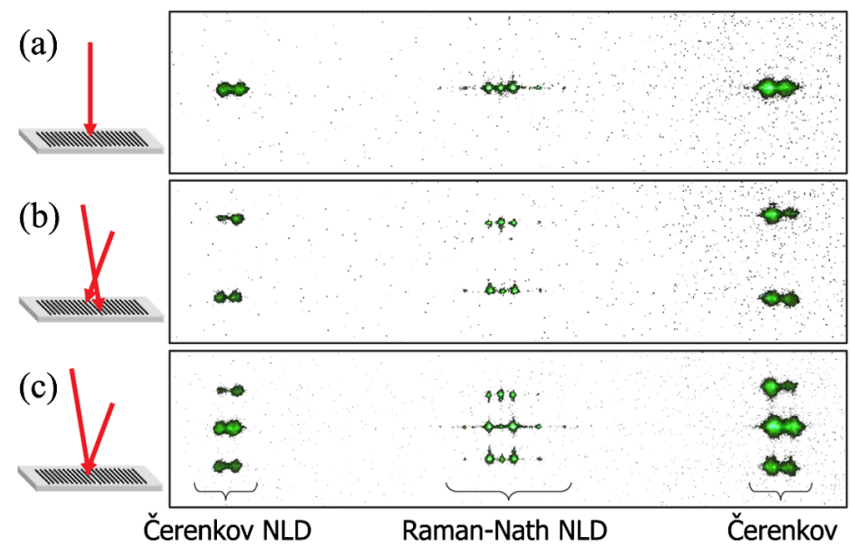

FIG. 2 (color online). SH patterns of Raman-Nath and Čerenkov NLD. (a) Single beam without a biprism, (b) with a biprism when both beams cross outside the sample, and (c) with a biprism when the beams cross inside the sample. noncollinear beams act as an equivalent beam directed along the bisector of the two fundamental beams, and this virtual (nonexistent) beam diffracts from the nonlinear grating creating its own set of diffraction spots. The angular position of these patterns coincides within our measurement error with those originating from a single beam.

The physics of the virtual-beam NLD can be understood directly from the transverse phase-matching condition shown in Fig. 3. The general vectorial phase-matching condition $\left(\vec{k}_{1 A}+\vec{k}_{1 B}\right)+m \vec{G}_{0}=\vec{k}_{2}$ can be split into two scalar conditions [13], namely, transverse, $k_{2} \sin \alpha_{m}=$ $m G_{0}$, and longitudinal, $k_{2} \cos \alpha=\left(k_{1 A}+k_{1 B}\right) \cos \gamma$. Here, $\alpha_{m}$ is the internal angle of the $m$ th diffraction order. It follows from these equations that the noncollinearity of the two fundamental beams affects only the longitudinal phase-matching conditions, while the transverse phase matching is not affected at all: it remains the same for both single or two fundamental beams. The SH NLD depends only on the transverse phase-matching conditions. Indeed, from the transverse phase-matching condition it follows that the internal diffraction angles are $\alpha_{m}=$ $\sin ^{-1}\left[m \lambda_{2} / n_{2} \Lambda\right]$, while the external angles are $\beta_{m}=$ $\sin ^{-1}\left[m \lambda_{2} / \Lambda\right]$.

The experimental values of the diffraction angles can be easily found by measuring the distance between the central spots $d$ and the distance between the diffraction patterns $p$, defined in Fig. 1(a). For the first-order diffraction we find the external angle $\beta_{1}=\tan ^{-1}\left[(d / p) \tan \gamma_{2 \omega}\right]$. In the same way, all higher-order angles can be found. We obtain $\beta_{m}=$ $2.13^{\circ}, 4.23^{\circ}, 6.32^{\circ}, 8.48^{\circ}, 10.64^{\circ}$ for $m=1,2,3,4$. These values coincide perfectly with the calculated $\beta_{m}=$ $2.11^{\circ}, 4.22^{\circ}, 6.34^{\circ}, 8.47^{\circ}, 10.61^{\circ}$ for a period $\Lambda=$ $14.3 \mu \mathrm{m}$. In addition, it can be seen in the images of

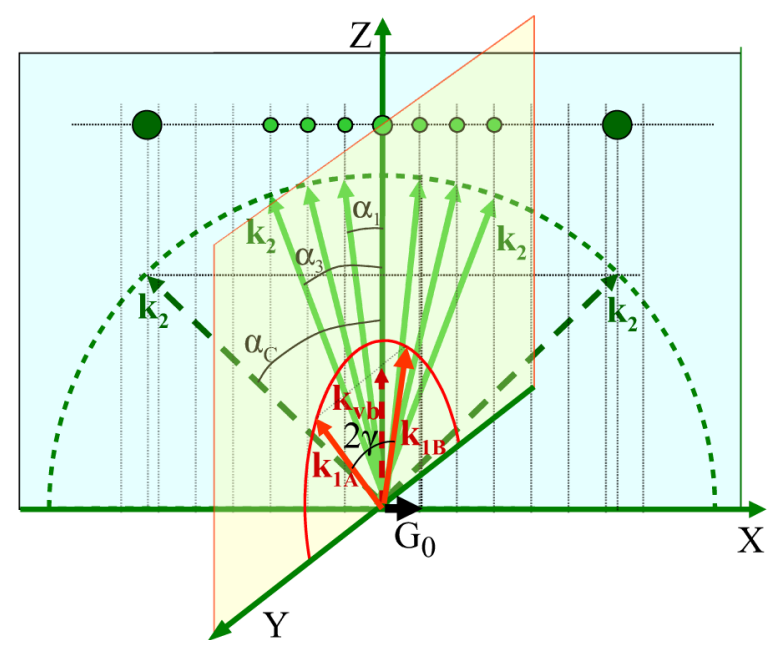

FIG. 3 (color online). Phase-matching diagram for RamanNath and Čerenkov NLD when both fundamental beams intersect inside the sample. All wave vectors of the SH beams, $\vec{k}_{2}$, lie in the $X-Z$ plane, while the wave vectors of the two fundamental beams, $\vec{k}_{1 A}, \vec{k}_{1 B}$, are located in the $Y-Z$ plane. $\vec{k}_{\mathrm{vb}}$ represents the wave vector of a virtual beam. 
Fig. 2 that the even diffraction orders are weaker than the odd orders. This is due to the fact that the duty cycle of the structure is close to 50\% and the even-order Fourier coefficients are very small.

The angular positions of the Čerenkov SH spots are slightly different for normal incidence of a single fundamental beam and for two noncollinear beams [see Fig. 2(c)]. In the single-beam excitation the two spots for the $E_{2}$ and $O_{2}$ interactions are found from the equations $\cos \alpha_{C, E 2}=2 k_{1} / k_{2, e}\left(\alpha_{C}\right)$ and $\cos \alpha_{C, O 2}=2 k_{1} / k_{2, o}$, where $k_{2, e}\left(\alpha_{C}\right)$ is the wave vector of the extraordinary polarized SH wave, propagating at Čerenkov angle $\alpha_{c}$. In the case of the biprism, the SH Cerenkov spots from the virtual beam [middle line in Fig. 2(c)] appear at slightly larger angles since $\cos \alpha_{C}$ will be smaller by the factor $\cos \gamma_{\mathrm{int}}$ :

$$
\begin{aligned}
& \cos \alpha_{C, O 2}=\left(k_{1 A}+k_{1 B}\right) \cos \gamma / k_{2, o}, \\
& \cos \alpha_{C, E 2}=\left(k_{1 A}+k_{1 B}\right) \cos \gamma / k_{2, e}\left(\alpha_{C}\right) .
\end{aligned}
$$

In Eq. (1) we can define a virtual beam with a wave vector $k_{v b}=\frac{1}{2}\left(k_{1 A}+k_{1 B}\right) \cos \gamma$. Derivation of Eq. (1) follows from Fig. 3. For a given SH polarization the Čerenkov SH spots generated by each individual beam and from the mixing between them have the same value of $\alpha_{C}$. For the (external) angular separation $\delta$ between the ordinary and the extraordinary polarized Čerenkov spots we obtain from Eq. (1) $\delta_{\text {theor }}=1.9^{\circ}$. This value agrees well with the experimentally measured value of $\delta_{\text {expt }}=1.8^{\circ}$.

Furthermore, we simulate numerically the nonlinear interaction of a single and two fundamental beams with the nonlinear periodic structure using a split-step Fourier method and assuming an undepleted pump approximation. The latter is well justified as the efficiency of the parametric process in our experiments remains of the order of $1 \%$. The fundamental beams are assumed to be Gaussian, each having a waist of $50 \mu \mathrm{m}$. These beams create nonlinear polarization source terms that are used to generate $\mathrm{SH}$ waves. The $\mathrm{SH}$ field at the output of the crystal is Fourier transformed, in order to explore its angular spectrum.

In Fig. 4 we display numerical results illustrating NLD on a one-dimensional $\chi^{(2)}$ grating and obtain good correspondence with our experiments. As can be seen, nonlinear Raman-Nath diffraction peaks appear at locations determined by the multiples of $G_{0}=2 \pi / \Lambda=4.3 \mu \mathrm{m}^{-1}$. At low spatial frequencies, only odd peaks are observed, since the duty cycle used in the simulations is exactly $50 \%$. The strong SH Čerenkov peaks appear for $G_{C}=77 \mu \mathrm{m}^{-1}$. This is close to, but not exactly, the 18th quasi-phasematching order $\left(G_{C} / G_{0}=17.9\right)$. The number $G_{C} / G_{0}$ is not integer because the position of the Cerenkov spots is defined solely by the longitudinal phase-matching condition, Eq. (1). In the numerical simulations shown in Fig. 4 only ordinary SH wave is considered, and this is the reason why, unlike the experiment (Fig. 2), additional Čerenkov $\mathrm{SH}$ spots do not appear.

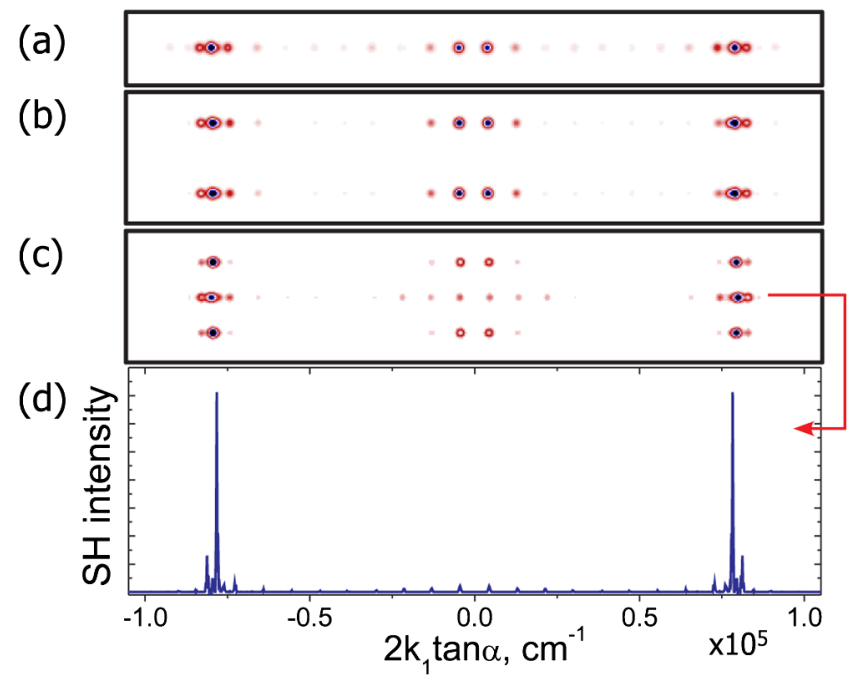

FIG. 4 (color online). Numerical results. (a) Diffraction from a single beam, (b) NLD from two beams crossing outside the sample, (c) NLD from two beams crossing inside the sample, and (d) SH intensity distribution for the virtual-beam NLD.

Finally, we test the applicability of the virtual-beam NLD to higher dimensional nonlinear periodic structures, and, in particular, to our annular periodically poled structure. The microphotograph of the $Z+$ surface of the structure is shown in Fig. 5(a). The schematic of this second experiment is sketched in Fig. 1(b) and a typical diffraction pattern is shown in Fig. 1(d). The two fundamental beams are created with the same biprism and they cross exactly in the center of the structure. We note that if the beams are shifted away from the center, the NLD will be similar to that from the one-dimensional quadratic grating. This simple correspondence enables the exact identification of the diffraction patterns.
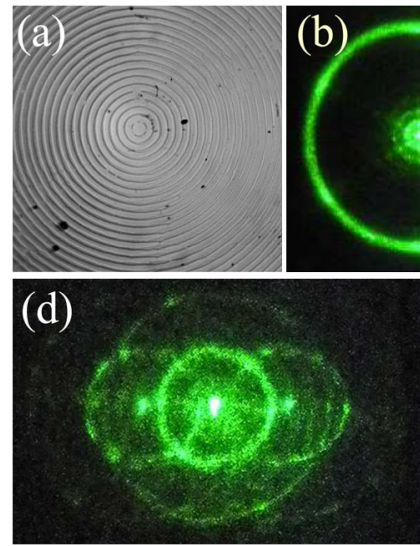

FIG. 5 (color online). (a) Microphotograph of the $Z$ surface of the annularly periodically poled structure. (b) Experimental image of NLD with a single fundamental beam and (c) with two beams crossing in the center of the structure. (d) Magnified central part of the pattern (c). (e) Numerically calculated $\mathrm{SH}$ emission from the virtual-beam NLD process. 
First, we excite the sample with a single laser beam [10]. In Fig. 5(b) we show the recorded SH pattern obtained at normal incidence of the beam (along axis $Z$ ). The internal rings result from the Raman-Nath NLD on the circular quadratic grating. Each ring corresponds to a conical wave with a characteristic angle defined by the relation $\sin \alpha_{m}=m G_{0} / k_{2}$, that corresponds to the transverse phase-matching condition. In this case (for normal incidence) the rings are centered around the axis $Z$ of the fundamental beam. Knowing the period of the annular grating and the wavelength, we obtain the following conical (external) angles for the first two diffraction rings: $\beta_{1}=4.02^{\circ}$ and $\beta_{2}=8.07^{\circ}$. The experimentally measured values are $4.0^{\circ}$ and $8.1^{\circ}$, respectively, and they coincide perfectly with our predictions. The strong external ring in Fig. 1(b) is due to the Cerenkov SH radiation $[11,12,14]$. This conical SH wave has a cone angle defined solely by the longitudinal phase-matching condition, $k_{2} \cos \gamma=2 k_{1}$.

Next, we study the NLD from two fundamental beams crossing in the center of the structure. Figures 5(c) and 5(d) show the experimentally recorded images of the NLD. The pattern consists of three sets of diffraction rings. The two side sets of rings result from the first-order single-beam NLD. On the other hand, the central rings (both 1st and 2nd orders are visible) result from two-beam interaction, and they appear only if the fundamental beams overlap in time and space in the center of the structure. The cone angles of the central rings do not depend on the biprism angle, and they are defined solely by Eq. (1). This is verified directly in the experiment. The reason is that the cone angles are determined by the transverse phase-matching condition, so that there is no difference in the ring diameters for both single or two-beam excitation.

The central bright spot in Fig. 5(d) is due to non-phasematched second-harmonic generation from the noncollinear mixing of both fundamental beams. The two side spots are non-phase-matched SH generated by each individual fundamental beam. The phase-matching diagram is the same as for the one-dimensional structures (Fig. 3). The brightest rings in the central part of Fig. 5(d) are due to the first-order diffraction. Every next higher-order ring is less intensive since it requires higher-order phase matching.

The bright Čerenkov SH ring [see Fig. 5(c)] comprises three different contributions. They include two rings originating from each fundamental beam and a third ring due to mixing of the two fundamental pulses. All these three conical SH signals are emitted at the same cone angle defined by Eq. (1), and they overlap in the case of close to normal incidence of the fundamental beam. As the birefringence of the stoichiometric lithium tantalate is small, the ordinary and extraordinary Čerenkov rings are indistinguishable in the experiment.
In Fig. 5(e) we illustrate the SH emission calculated for the annular structure in SLT. The agreement with the experiment is excellent. Note the azimuthal intensity modulation of the side rings seen in both the experimental image [Fig. 5(d)] and in the simulations [Fig. 5(e)]. This effect is a manifestation of Maker fringes which arise because these $\mathrm{SH}$ signals are generated in a non-phasematching regime (only the transverse phase-matching condition is satisfied).

In conclusion, we have observed a novel wave phenomenon of nonlinear diffraction produced by a virtual fundamental beam in one- and two-dimensional periodic quadratic gratings. We have demonstrated that the angular positions of the diffracted maxima can be explained by either longitudinal and/or transverse phase-matching conditions. This type of nonlinear diffraction requires simultaneous interaction of two fundamental beams and has no analogy in linear wave physics. Importantly, the observed effect is not restricted to optics, and it can be observed in other settings such as nonlinear diffraction of acoustic waves in periodic structures or matter waves in optical lattices. In optics, this effect can find applications in second-harmonic microscopy as well as in testing the parameters of the periodically poled structures (e.g., period and duty cycle) with no restriction on the input wavelength and without surface etching.

The work was supported by Australian Research Council and Israeli Science Foundation (Grant No. 960/05).

*Deceased.

[1] M. Born and E. Wolf, Principles of Optics (Cambridge University Press, Cambridge, England, 1999).

[2] P. Kapitza and P. A. M. Dirac, Proc. Cambridge Philos. Soc. 29, 297 (1933); H. Batelaan, Contemp. Phys. 41, 369 (2000).

[3] H. Mueller, S. W. Chiow, and S. Chu, Phys. Rev. A 77, 023609 (2008).

[4] A. D. Cronin, J. Schmiedmayer, and D. E. Pritchard, Rev. Mod. Phys. 81, 1051 (2009).

[5] I. Freund, Phys. Rev. Lett. 21, 1404 (1968).

[6] G. Dolino, J. Lajzerow, and M. Vallade, Phys. Rev. B 2 , 2194 (1970).

[7] Y. Le Grand et al., Opt. Commun. 200, 249 (2001).

[8] S. M. Saltiel et al., Opt. Lett. 34, 848 (2009).

[9] D. Kasimov et al., Opt. Express 14, 9371 (2006).

[10] S. M. Saltiel et al., Phys. Rev. Lett. 100, 103902 (2008).

[11] S. M. Saltiel et al., IEEE J. Quantum Electron. 45, 1465 (2009).

[12] S. J. Holmgren, C. Canalias, and V. Pasiskevicius, Opt. Lett. 32, 1545 (2007).

[13] S. P. Tewari, H. Huang, and R. W. Boyd, Phys. Rev. A 54, 2314 (1996).

[14] A. Zembrod, H. Puell, and J. Giordmaine, Optoelectronics 1, 64 (1969). 